\title{
Time Series Analysis on the Ratio For Pixels With Abnormal Brightness Temperature Increase and Its Variation Before Some Earthquakes with MS $\geq 5.0$ in the Taiwan Area
}

by Liu Fang

\author{
Earthquake Administration of Sichuan Province, Chengdu 610041, China
}

\section{Abstract}

In the study of application of MODIS satellite remote sensing data to earthquake prediction, the paper puts forward for the first time a quantitative method to estimate the ratio for the pixels with abnormal brightness temperature (BT) increase and a preliminary scheme for cloud removal. The principle is that, firstly, the cloudless data observed by the same satellite at the same period of time but in different days (usually 1 to 3 days) are mosaicked to get high cloudless rate data, and then the BT variation curve and mean square difference (MSD) of each pixel are calculated with the data from the covered area to determine daily whether the BT data of the day is normal or not at certain pixel by using twice of MSD as criterion. The ratio for the pixels with abnormal BT increase can be calculated by dividing the total number of abnormal pixels with the total pixels of the whole area. Analysis on a series of recent earthquakes in Taiwan area shows that the ratio for pixels with abnormal BT increase, which normally undulates around zero, has a sudden enhancement 1 to 20 days before the medium-strong earthquakes. It is expected that a new method for identifying earthquake auspice could be found through special studies in regions with frequent seismic activity by analyzing the change of the ratio for the pixels with abnormal BT increase from MODIS satellite remote sensing infrared (IR) information from which the effect of cloud has been removed to a certain extent.

(1)The area ratio between Area I and Area II is $3.5=1$, and the ratio of ocean area to the land area in Area I is $2.5=1$. It means that the BT data quantity of the sea surface is 2.5 times of that of land. However, the morphology of the curves of BT anomaly variation with time in Fig. 3 and Fig.4 is basically the same, except the difference in amplitude. Despite the fact that the amount of sea surface BT data is 2.5 times of the land's, it does not create big difference in the curves between the two areas. This shows that the variation of ratios of BT anomaly obtained in this paper results mainly from the BT change in the land and the adjacent sea areas (see the sketch map of Area II in Fig. 1). All the 14 earthquakes of $M s \geq 5.0$ studied in this paper occurred in this area. So it can be concluded that the BT anomaly ratio discussed in the paper embodies the anomaly information relating to earthquake to a certain extent. As one of the seismic anomaly identification methods, it can be helpful in finding out the occurrence of BT anomaly in certain period of time so as to raise our attention and to go into it for its possible causes. As for whether all the BT anomaly is related to earthquake, we need further studies.

(2) In our research in the paper, we tried to remove the effect of annual variation by polynomial fitting of MODIS data of the recent two years but did not get ideal effects. The authors investigated the effectiveness for the inland area and the results showed that the polynomial fitting of MODIS data is an effective method for the removal of annual effect. It seems to suggest that there exists a big difference in the environment for the detected pixels between the coast area and the inland, and therefore it will be improper to use the same method in the markedly different environments. In calculating and processing satellite thermal IR remote sensing information, we shall take into account both the general and the specific characters of data processing in different study regions.

(3) According to the ratio of the pixels with BT anomaly, we can plot out the corresponding spatial distribution of BT anomaly (the paper does not give the spatial anomaly distribution for each abrupt enhancement of the ratio due to limitation of paper length). The spatial distributions do not show an obvious migration of BT anomaly areas towards the epicenters. The reason for this is maybe that the variations of ratio anomalies in Fig. 3 and Fig. 4 are all expressed by abrupt enhancements; so, generally they did not show any continuity. Meanwhile, we can also find from the anomaly's spatial distribution that not all earthquakes occurred in the BT anomaly area. So, though the variation of the ratio of pixels with abnormal BT increase reveals a certain degree of correlation with earthquake, we can not estimate the location of epicenter relying only on the variation of BT anomaly ratio.

(4) The red lines in Fig.3 and Fig.4 are thresholds of ratio for pixels with BT anomaly. The threshold for Area I is 0.5 , and that for Area II is 1.4. It should be noted that the determination of threshold is non-unique. The significance of the threshold is to set up a quantitative criterion for discriminating the abrupt enhancement of the ratio. Therefore, the principle for determining the threshold shall refer to the maximum relevancy of all earthquakes in the study time interval.

By analyzing the relation of the variation of ratio of the pixels with abnormal BT increase with moderate strong earthquakes in Taiwan area, we can come to the following conclusions: with MODIS satellite thermal IR remote sensing data, in which the cloud effect has been removed to a certain extent, we can derive the variations of the ratio of pixels with abnormal BT increase, and basing on this, we can expect to find out a new method for identifying the short-term and imminent earthquake precursors through specific and systematic researches in earthquake-prone areas. The physics behind this ratio variation of pixels with BT anomaly and its inherent connection with earthquake preparation need to be further investigated. It is suggested that while doing the theoretical research, we shall promote the research on the short-term and imminent earthquake monitoring and analysis by using efficiently the MODIS thermal IR remote sensing information in specific regions and environments based on the recognized phenomena, and we are sure that this can be of a great help for earthquake prevention and disaster reduction.

Keywords: MODIS; Satellite thermal infrared data; Ratio for pixels with abnormal BT increase; Earthquake. 